\title{
A Rare Case of Ulnar and Popliteal Artery Thrombosis in Antiphospholipid Antibody Syndrome
}

\author{
Ashish Kumar Nayak', Debasish Das² \\ Departments of ${ }^{1}$ Dermatology and ${ }^{2}$ Cardiology, All India Institute of Medical Sciences, Bhubaneswar, Odisha, India \\ ORCID: \\ Ashish Kumar Nayak: 0000-0003-2056-9117 \\ Debasish Das: 0000-0002-6228-9554
}

\section{Abstract}

We report an extremely rare case of medium vessel thrombosis (ulnar and popliteal artery) in a 26-year-old female presenting with recurrent abortion and gangrenous right ring finger and pregangrenous toes of the left feet. Medium vessel thrombosis i.e. selective involvement of ulnar and popliteal vessels is an extremely rare phenomenon described in the literature of antiphospholipid antibody (APLA) syndrome. We conservatively managed the patient with antiplatelets and anticoagulants which substantially improved the ischemia of the finger and toes. Our case is a unique description of ulnar and popliteal artery thrombosis in APLA syndrome which has not been described in world literature so far.

Keywords: Antiphospholipid antibody syndrome, artery, popliteal, ulnar

\section{INTRODUCTION}

Antiphospholipid syndrome (APS) is an acquired autoimmune disorder that manifests as recurrent venous or arterial thrombosis with or without fetal loss. ${ }^{[1]}$ The most common differential diagnosis is lupus anticoagulant (LA) syndrome which manifests with thrombotic rather than hemorrhagic complications. APS can be primary or secondary in association with systemic lupus erythematosus (SLE), rheumatic, or autoimmune disorder. Currently, the preferred terminology is APS with or without the associated rheumatic disease. Although antiphospholipid antibody (aPL) antibodies are clinically linked with APS, whether they have a role in the pathogenesis or are an epiphenomenon is still not clear because up to $5 \%$ of healthy individuals are known to have aPL antibodies. Interestingly, the development of aPL antibodies has been described in association with thrombosis in patients with COVID-19[2] seropositive asymptomatic individuals who do not require specific treatment. Prophylaxis is needed during surgery, hospitalization, pregnancy, or with associated autoimmune disease. Although low-dose aspirin had been

Received: 26-05-2021 Revised: 17-07-2021 Accepted: 18-07-2021

Published Online: 25-09-2021

\begin{tabular}{|l|l|}
\hline \multicolumn{3}{|c|}{ Access this article online } \\
\hline Quick Response Code: & Website: \\
& \\
http://www.ijcva.com
\end{tabular}

widely used, its effectiveness remains unproven. As noted in our case, full anticoagulation with heparin followed by warfarin therapy during thrombotic episodes recovers most of the patients with APLA.

\section{Case Report}

A 26-year-young female presented to the dermatology outpatient department with the chief complaint of blackening of the right ring finger [Figure 1] and paleness of the toes of the left foot [Figure 2] with the left calf and right forearm claudication. On examination, she had a blood pressure of $110 / 70 \mathrm{~mm} \mathrm{Hg}$ in the right arm supine position with a pulse rate of 80 beats per min. Her cardiac system evaluation was within the normal limit and examination of peripheral pulses revealed absent left popliteal, right ulnar with the diminished pulsation of left posterior tibial, and dorsalis pedis artery. Her serum chemistry including complete blood count, sugar profile,

Address for correspondence: Dr. Debasish Das, Department of Cardiology, All India Institute of Medical Sciences, Bhubaneswar - 751 019, Odisha, India. E-mail: dasdebasish54@gmail.com

This is an open access journal, and articles are distributed under the terms of the Creative Commons Attribution-NonCommercial-ShareAlike 4.0 License, which allows others to remix, tweak, and build upon the work non-commercially, as long as appropriate credit is given and the new creations are licensed under the identical terms.

For reprints contact: WKHLRPMedknow_reprints@wolterskluwer.com

How to cite this article: Nayak AK, Das D. A rare case of ulnar and popliteal artery thrombosis in antiphospholipid antibody syndrome. Int J Cardiovasc Acad 2021;7:106-9. 


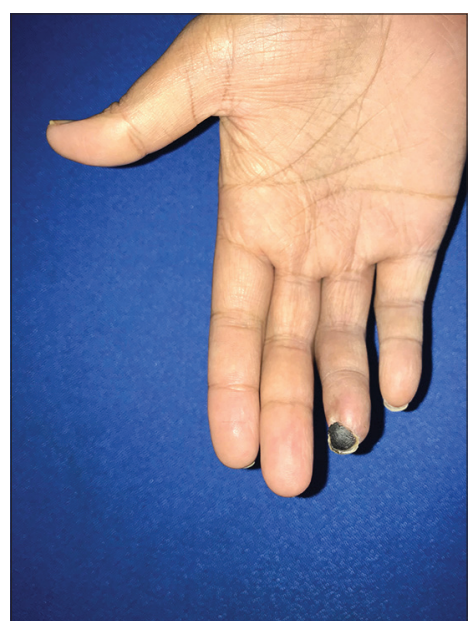

Figure 1: Gangrene of right ring finger

lipid panel, and thyroid profile was within the normal limits. Her erythrocyte sedimentation rate was $52 \mathrm{~mm} / \mathrm{h}$. She revealed spontaneous abortion of the first baby in the late first trimester 3 years back. Her ultrasonography (USG) abdomen and pelvis were within the normal limit. Baseline electrocardiogram and echocardiography were within the normal limit. Duplex USG revealed loss of triphasic flow pattern in the right ulnar and left popliteal artery. Then she was advised for the right forearm and aortobifemoral computed tomography (CT) angiography which revealed completely occlusive thrombus in the left popliteal artery [Figure 3] and thrombotic occlusion of the right ulnar artery [Figure 4]. Because of the gangrenous right ring finger with pregangrenous toes along with a history of abortion, we thought of antiphospholipid antibody syndrome and sent for an APLA profile. Immunoglobulin $\mathrm{G}(\mathrm{IgG})$ cardiolipin was elevated i.e. $35 \mathrm{U}$ (<12 units), immunoglobulin M (IgM) cardiolipin was elevated i.e. $218 \mathrm{U}$ ( $<12$ units), immunoglobulin A (IgA) $\beta_{2}$ microglobulin was elevated with the value of 96 units, $\operatorname{IgG} \beta_{2}$ icroglobulin was elevated with the value of 108 units $(<12)$, IgM $\beta_{2}$ microglobulin was elevated with the value of 273 units, and LA was positive with Dilute Russell's viper venom time (DRVVT) (Russell Viper Venom Test) test ratio of 2.67 (1.3). Her antinuclear antibody (ANA) was moderately raised with a mixed pattern of distribution (homogeneous and specked). Her ANA by immunofluorescence assay method revealed anti-ds DNA and anti-RNP/Sm antibody to be positive and ANA by enzyme-linked immunosorbent assay revealed anti-histone and anti-nucleosome antibody to be positive. We diagnosed the patient as anti-phospholipid antibody syndrome with elevated anticardiolipin (aCL) antibodies and positive LA. We managed the patient with extended heparin therapy with Inj low molecular weight heparin $60 \mathrm{mg}$ subcutaneous twice daily for 8 days with baby aspirin $75 \mathrm{mg}$ once daily and warfarin $2 \mathrm{mg}$ once daily. We monitored the daily prothrombin time international normalized ratio (INR) and kept it between 2 and 3. The patient's ischemic toes substantially improved with no recurrence of calf claudication or the right forearm claudication and the color of the toes became normal. Because of the resolution of symptoms, we did not proceed for left

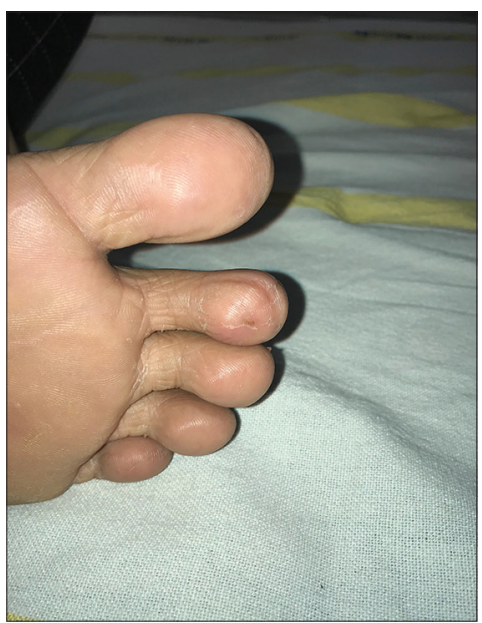

Figure 2: Pregangrenous toes of the feet

popliteal plain balloon angioplasty and revascularization of the right ulnar artery. The patient well responded to antiplatelets and anticoagulants. Our case is a unique demonstration of medium vessel, i.e. ulnar and popliteal thrombosis in aPL syndrome which has not been described in the literature so far, managed with antiplatelets and anticoagulants without the need of peripheral arterial intervention.

\section{Discussion}

It is estimated that the incidence of APS is approximately five cases per 100,000 persons per year, and the prevalence is approximately 40-50 cases per 100, 000 persons. ${ }^{[3]}$ Antiphospholipid antibodies are found in approximately $30 \%-40 \%$ of the patients with SLE. ${ }^{[4]}$ Mechanisms of thrombosis in APS is due to defect in cellular apoptosis, which exposes membrane phospholipids which bind beta-2 glycoprotein I and this phospholipid-protein complex with a neoepitope become the target of autoantibodies. Other proposed mechanisms include the production of antibodies against prothrombin, protein $\mathrm{C}$, protein $\mathrm{S}$, and annexins, and activation of platelets and vascular endothelium. The role of complement activation has been increasingly recognized as a primary event in pregnancy loss. ${ }^{[5,6]}$ Hypercoagulability and recurrent thrombosis present with deep venous thrombosis, stroke, sinus thrombosis, seizures, chorea, and reversible cerebral vasoconstriction syndrome. Interestingly, our case presented with medium vessel arterial thrombosis involving popliteal and ulnar artery which has not yet been described in the literature. A "two-hit" theory has been proposed in which a second risk factor (age, hypertension, diabetes, obesity, smoking, pregnancy, and surgery) incites the thrombotic effects of aPL. ${ }^{[7]}$ Interestingly, the patient did not have any of the secondary risk factors mentioned above. Triple therapy (anticoagulation, corticosteroids, plasma exchange, and/or intravenous immunoglobulin) improves complications of APS. Late spontaneous fetal loss (second or third trimester) is common in APS; however, it can occur at any time during pregnancy as in our case it was noted in the first trimester. 


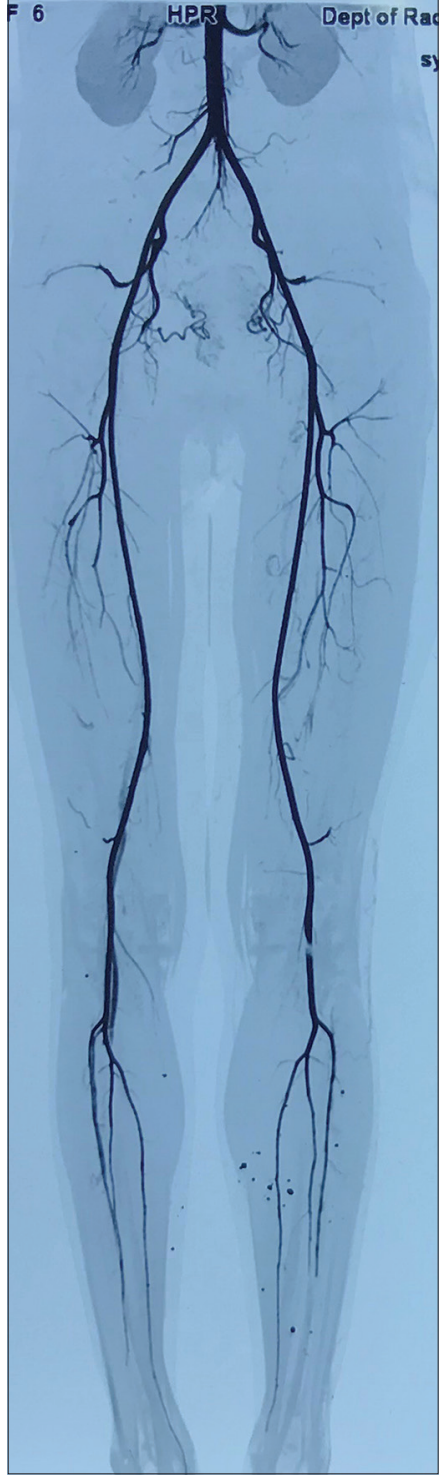

Figure 3: Thrombus in mid popliteal artery

In 2006, revised criteria for the diagnosis of APS defined at least one clinical criterion and one laboratory criterion must be present for a patient to be classified as having APS. ${ }^{[8]}$ The clinical criteria consist of vascular thrombosis and pregnancy morbidity. Vascular thrombosis is defined as one or more clinical episodes of arterial, venous, or small-vessel thrombosis in any tissue or organ confirmed by findings from imaging studies, Doppler studies, or histopathology. Thrombosis in a young individual (males $<55 \mathrm{y}$; females $<65 \mathrm{y}$ ) or in the absence of conventional risk factors warrant APS evaluation. Pregnancy morbidity is defined as the following: one or more late-term ( $>10$ weeks' gestation) spontaneous abortions, one or more premature births ( $<34$ weeks) due to severe preeclampsia, eclampsia, or placental insufficiency, and three or more spontaneous abortions before 10 weeks gestation. Laboratory criteria include any of the following; raised level of IgG or IgM aCL, anti-beta-2 glycoprotein I, and LA on at least two occasions minimum 12 weeks apart. APLA is found

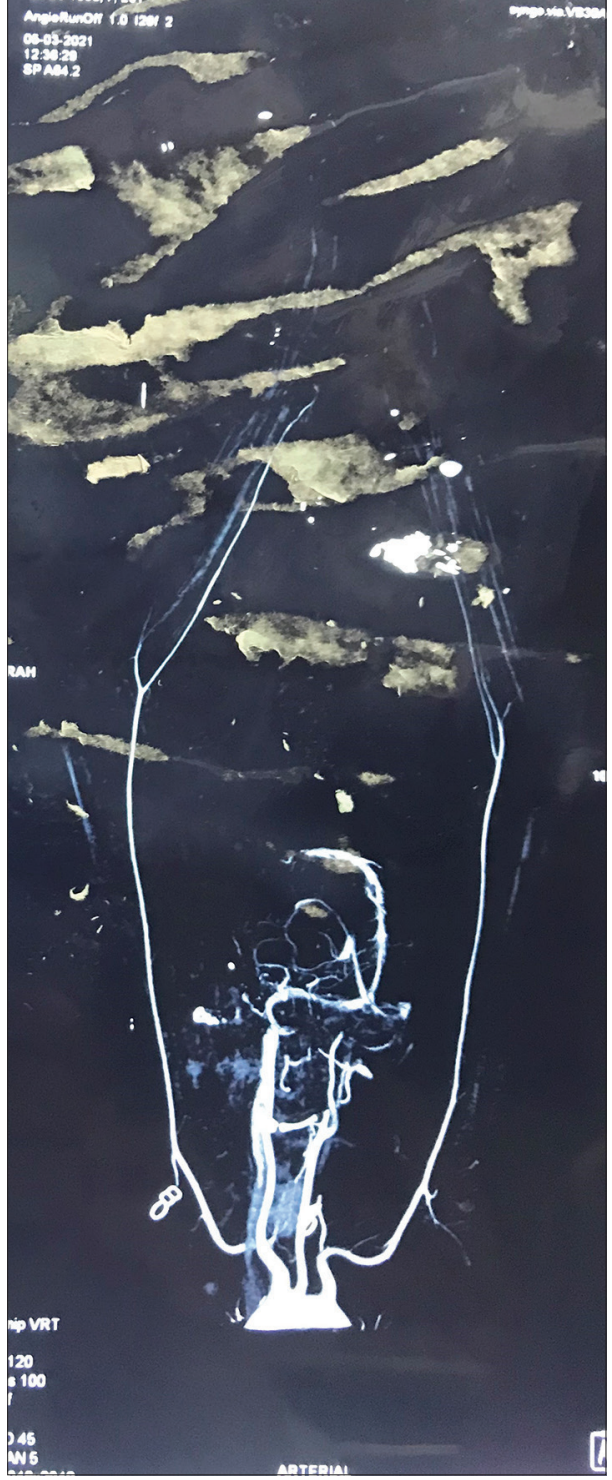

Figure 4: Thrombus in right ulnar artery

in SLE (25\%-50\%), Sjogren syndrome (42\%), rheumatoid arthritis (33\%), autoimmune thrombocytopenic purpura $(30 \%)$, psoriatic arthritis (28\%), systemic sclerosis (25\%), mixed connective tissue disorder (22\%), giant cell arteritis (20\%), and in Behcet's disease (20\%). ${ }^{[9]}$ Common differential diagnosis of APS includes hypercoagulable states such as malignancy, oral contraceptive use, hormone replacement therapy, homocysteinemia, antithrombin III deficiency, protein $\mathrm{C}$ or $\mathrm{S}$ deficiency, factor $\mathrm{V}$ Leiden mutation, prothrombin A mutation, antiprothrombin antibodies, atherosclerotic vascular disease, including multiple cholesterol emboli syndrome, and systemic necrotizing vasculitis. Common differential diagnoses include disseminated intravascular coagulation, infective endocarditis, and thrombotic thrombocytopenic purpura (TTP). Out of the 3 known isotypes of aCL (i.e. IgG, $\operatorname{IgM}, \operatorname{IgA}$ ), $\operatorname{IgG}$ correlates most strongly with thrombotic events. Cardiolipin is the dominant antigen used in most serologic tests for syphilis; consequently, these patients may have a false-positive test 
result for syphilis as noted in our case. The presence of LA is confirmed by mixing normal platelet-poor plasma with the patient's plasma. If a clotting factor is deficient, the addition of normal plasma corrects the prolonged clotting time. If the clotting time does not normalize, then an inhibitor or LA is present. "Triple-positive" patients (LA, anti-beta-2 glycoprotein antibodies, and AC antibodies) are at highest risk for thrombosis or abnormal pregnancy, and possibly for recurrence as noted in our case who developed thrombosis of medium vessels. ${ }^{[7]}$ Thrombocytopenia and paradoxical thrombosis are fairly common in patients with APS $(22 \%$ at presentation, 30\% cumulatively). Full anticoagulation with heparin followed by warfarin therapy is administered in APS-related thrombosis with a target INR of 2.0-3.0 for venous thrombosis and 3.0 for arterial thrombosis. Direct thrombin inhibitors and factor Xa inhibitors such as rivaroxaban have a role in warfarin intolerance/allergy or poor control of $\mathrm{INR}^{[10]}$ as concluded from rivaroxaban for antiphospholipid Syndrome trial. ${ }^{[1]}$ Rituximab has a role in noncriteria aPL manifestations including thrombocytopenia and skin ulcers. ${ }^{[7]}$ Pregnancy with APS is treated with subcutaneous heparin and baby aspirin antepartum and 6 weeks postpartum. ${ }^{[12]} \mathrm{An}$ inferior vena cava (IVC) filter may have a role in anticoagulation intolerant patients or who develop thrombosis despite maximum anticoagulation. IVC filter is contraindicated during acute APS due to increased risk of IVC thrombosis and pulmonary embolism. Activated partial thromboplastin time (aPTT) is unreliable in the presence of aPL antibodies which elevate aPTT. Hence, factor Xa may be helpful in this setting for monitoring anticoagulation. Catastrophic APS (CAPS) is conventionally treated with plasma exchange, intravenous immunoglobulins, and corticosteroids in addition to full anticoagulation. ${ }^{[13]}$ Eculizumab, a humanized monoclonal antibody against $\mathrm{C} 5$ complement protein, may be helpful in CAPS, and postrenal transplant patients. ${ }^{[14]}$ The use of corticosteroids is reserved for nonthrombotic manifestations such as associated thrombocytopenia, autoimmune hemolytic anemia, or the treatment of an underlying connective tissue disease. The annual incidence of the first thrombosis in APS is approximately $0 \%-5 \% \cdot{ }^{[15]}$ Interestingly, patients with secondary APS have a similar prognosis as compared with primary APS. We managed the patient successfully with extended heparin therapy for 8 days with aspirin and warfarin therapy which improved the pregangrenous condition of the skin with the resolution of limb claudication.

\section{Conclusion}

Our case is unique and the first to describe medium vessel thrombosis of the ulnar and popliteal artery in APS in a young woman which has not been described in the literature so far. It was successfully managed with aspirin, extended low molecular weight heparin, and anticoagulant. Limb gangrene may not be always secondary to peripheral arterial disease, an underlying APS should always be thought of in a young female presenting with recurrent abortion. In time and prolonged medication avoids extreme complications and improves the morbidity and mortality in APS.

\section{Declaration of patient consent}

The authors certify that they have obtained all appropriate patient consent forms. In the form the patient(s) has/have given his/her/their consent for his/her/their images and other clinical information to be reported in the journal. The patients understand that their names and initials will not be published and due efforts will be made to conceal their identity, but anonymity cannot be guaranteed.

\section{Financial support and sponsorship}

Nil.

\section{Conflicts of interest}

There are no conflicts of interest.

\section{REFERENCES}

1. Sammaritano LR. Antiphospholipid syndrome. Best Pract Res Clin Rheumatol 2020;34:101463.

2. McFadyen JD, Stevens H, Peter K. The emerging threat of (Micro) thrombosis in COVID-19 and its therapeutic implications. Circ Res 2020;127:571-87.

3. Gómez-Puerta JA, Cervera R. Diagnosis and classification of the antiphospholipid syndrome. J Autoimmun 2014;48-49:20-5.

4. Lockshin MD. Update on antiphospholipid syndrome. Bull NYU Hosp Jt Dis 2008;66:195-7.

5. Giannakopoulos B, Krilis SA. The pathogenesis of the antiphospholipid syndrome. N Engl J Med 2013;368:1033-44.

6. Girardi G, Redecha P, Salmon JE. Heparin prevents antiphospholipid antibody-induced fetal loss by inhibiting complement activation. Nat Med 2004;10:1222-6.

7. Nalli C, Andreoli L, Casu C, Tincani A. Management of recurrent thrombosis in antiphospholipid syndrome. Curr Rheumatol Rep 2014;16:405.

8. Miyakis S, Lockshin MD, Atsumi T, Branch DW, Brey RL, Cervera R, et al. International consensus statement on an update of the classification criteria for definite antiphospholipid syndrome (APS). J Thromb Haemost 2006;4:295-306.

9. Cohen D, Berger SP, Steup-Beekman GM, Bloemenkamp KW, Bajema IM. Diagnosis and management of the antiphospholipid syndrome. BMJ 2010;340:c2541.

10. Erkan D, Aguiar CL, Andrade D, Cohen H, Cuadrado MJ, Danowski A, et al. $14^{\text {th }}$ International Congress on Antiphospholipid Antibodies: Task force report on antiphospholipid syndrome treatment trends. Autoimmun Rev 2014;13:685-96.

11. Cohen H, Hunt BJ, Efthymiou M, Arachchillage DR, Mackie IJ, Clawson S, et al. Rivaroxaban versus warfarin to treat patients with thrombotic antiphospholipid syndrome, with or without systemic lupus erythematosus (RAPS): A randomised, controlled, open-label, phase 2/3, non-inferiority trial. Lancet Haematol 2016;3:e426-36.

12. Committee on Practice Bulletins - Obstetrics; American College of Obstetricians and Gynecologists. Practice bulletin No. 132: Antiphospholipid syndrome. Obstet Gynecol 2012;120:1514-21.

13. Mar N, Kosowicz R, Hook K. Recurrent thrombosis prevention with intravenous immunoglobulin and hydroxychloroquine during pregnancy in a patient with history of catastrophic antiphospholipid syndrome and pregnancy loss. J Thromb Thrombolysis 2014;38:196-200.

14. D'Cruz D. Renal manifestations of the antiphospholipid syndrome. Curr Rheumatol Rep 2009;11:52-60.

15. Cervera R. Antiphospholipid syndrome. Thromb Res 2017;151 Suppl 1:S43-7. 